\title{
Hilbert Based Testing of ADC Differential Non-linearity Using Wavelet Transform Algorithms
}

\author{
Emad A. Awada \\ Electrical and Computer Engineering, Applied Science Private University, Jordan
}

\begin{tabular}{l} 
Article Info \\
\hline Article history: \\
Received Dec 25, 2017 \\
Revised Jul 28, 2018 \\
Accepted Aug 11, 2018 \\
\hline
\end{tabular}

\section{Keyword:}

Differential Non-Linearity Digital-to-Analog Converters Discrete Wavelet Transform Hilbert Transform

\begin{abstract}
In testing Mixed Signal Devices such as Analog to Digital and Digital to Analog Converters, some dynamic parameters, such as Differential NonLinearity and Integral Non-linearity, are very critical to evaluating devises performance. However, such analysis has been notorious for complexity and massive compiling process. Therefore, this research will focus on testing dynamic parameters such as Differential Non-Linearity by simulating numerous numbers of bits Analog to Digital Converters and test the output signals base on new testing algorithms of Wavelet transform based on Hilbert process. Such a new testing algorithm should enhance the testing process by using less compiling data samples and prompt testing results. In addition, new testing results will be compared with the conventional testing process of Histogram algorithms for accuracy and enactment.
\end{abstract}

Copyright $\odot 2018$ Institute of Advanced Engineering and Science. All rights reserved.

\section{Corresponding Author:}

Emad A. Awada,

Department of Electrical and Computer Engineering,

Applied Science Private University, Amman, Jordan.

Email: e_awada@asu.du.jo

\section{INTRODUCTION}

The notion of converting analog to digital waveform (digitizing) is very critical task for Digital Signal Processing (DSP) applications to enable gathering, analyzing, and interpolating analog data in digital domain. However, this conversion of analog to digital representation requires mixed signal Analog to Digital Converters (ADCs), which comes in many types and speed for fast and instantaneous digitizing process. Though, to ensure the proper performance of DSP devices, thorough testing parameters are required to eliminate ADCs false representation outputs. For example, parameter such as Differential Non-Linearity (DNL) must be checked for any abnormality output characteristics due to error added or deviation from the original analog waveform [1], [2].

As a result, such testing will ensure proper performance of applications as expected. However, at manufacture level, testing of such devicescan be very lengthy and complicated especially with higher number of bits ADCs [2]. For instance, in [1]-[3], conventional testing of such parameters requires large number of data samples which make testing and compiling process very tedious task and lengthy process. That is, all output waveform data codes need to be included in compiling process and compared with ideal codes for DNL. Each code contains a large number of samples that forming a fixed length of codes known as Least Significant Bits (LSB) [2], [4].

Therefore, an increase in ADCs number of bits results in code increases by $2^{\mathrm{n}}$ ( $\mathrm{n}$ is number of ADC bits) and increase number of data samples making the testing process very lengthy. In addition, conventional methods of testing waveform characteristics such as FFT and Sinusoidal Histogram have been known to include error in testing compile process and estimation results [2], [6]-[8]. For instance, while FFT is based on additive noise model and require a large number of samples bit [1], [2], [9], [10], Sinusoidal Histogram majority of samples collected are localized at both ends of the Histogram to produced larger error near the peaks [2]. Therefore, deviation produced by faulted bits or external error will be included in estimating code 
length.As a result, testing process must be enhanced to shorten testing period of time with importance of high sensitivity of data analysis.

Large number of researches has investigated classical testing algorithms to enhance testing algorithms. While some works have performed in the area of Fourier Transform and Sinusoidal Histogram [11]-[14], others have investigated new testing algorithms such as Wavelet transforms [1], [2], [9], [15], [16]. While, In previous works, [2]-[5], DNL errors for numerous ADC devices have been tested using Wavelet transform, this work will focus on the benefit of combining Hilbert Transform and Wavelet transform to shorten testing time significantly by reducing the total number of required collected data samples. That is, using Hilbert Transform as a base signal module and Wavelet transform as a data extracting processto achieve faster testing process and of higher testing sensitivity of bits error analysis.

\section{THEROTICAL BACKGROUND}

Ideally, to measure ADCs output waveform in term of DNL, the device under test (ADCs) output voltage range need to be determined for step size estimation (Least Significant Bit (LSB)) (1). That is, the device output full voltage range (Full Scale Rang (FSR)) is divided into equal segments of voltage. Each division will contain a specific range of voltage values

$$
1 L S B=\frac{\text { Voltage }\left[2^{n}-1\right]-\operatorname{Voltage}[0]}{2^{n}-1}
$$

Where $\mathrm{n}$ is ADC number of bits

Segments deviation can be measure among consecutive codes of LSB. Therefore, DNL can be determinate by (2)

$$
D N L=\frac{V[i+1]-V[i]}{L S B}-1
$$

\section{TESTING METHODOLOGY}

In this simulation of testing ADCs DNL, testing is based on generating digital waveform codes to measures output corresponding voltages. As shown in [16], by capturing output voltage code, Hilbert Transform and Wavelet decomposition will be applied respectively to transfer signal and eliminate extra unrequired data.

In this proposed testing algorithms, Hilbert Transform is used to define the real and imaginary parts of a signal (3). Such a process will enhance the ability to detect function peak through interpolation [16], [17]. As a result, the computation of waveform samples can be based on one part of divide complex signal.

$$
\mathrm{z}(\mathrm{t})=\mathrm{g}(\mathrm{t})+\mathrm{j} \tilde{g}(t)
$$

However, in this work, the imaginary part of tested waveform $\hat{g}[t]$ will be collected (a summation of input cosine wave and other non-input components (noises $\varepsilon[n]$ ) (4).

$$
\hat{g}[t]=g[n]+\varepsilon[n]
$$

and the modulated output waveform can be define as in (5)

$$
\tilde{\hat{g}}[m]=A \sin \left(2 \pi f_{0} n+\phi\right)+\varepsilon[m]
$$

Yet, modulated signal will undergo further decomposition and filtering process by implementing Discrete Wavelet Transform (DWT). With two special properties of convolution and down-sampling process, Wavelet transform can produce a unique algorithm in analyzing the output tested waveform data. Assuming a discrete signal $\left(\mathrm{s}_{\mathrm{n}}=\left\{\mathrm{s}_{\mathrm{nk}}\right\}\right)$, Wavelet decomposition for approximation and detailing coefficient can be obtained respectively by (6) and (7) respectively 


$$
\begin{aligned}
& s_{n-1, j}=\sum_{k} \tilde{h}_{k-2 j} s_{n k} \\
& d_{n-1, j}=\sum_{k} \tilde{g}_{k-2 j} s_{n k}
\end{aligned}
$$

This process is done by convolution as shown (8) and (9)

$$
\begin{aligned}
& \left(\tilde{h}(-) * s_{n}\right)_{j}=\sum_{k} \tilde{h}_{-(j-k)} s_{n k} \\
& \left(\tilde{g}(-) * s_{n}\right)_{j}=\sum_{k} \tilde{g}_{-(j-k)} s_{n k}
\end{aligned}
$$

Followed by down-sampling as in (10) and (11)

$$
\begin{aligned}
& s_{n-1}=(\downarrow 2)\left(\tilde{h}(-) * s_{n}\right) \\
& d_{n-1}=(\downarrow 2)\left(\tilde{g}(-) * s_{n}\right)
\end{aligned}
$$

As a result, number of processed data samples will be reduced by half (half the bandwidth) starting from the largest energy level and down into a sub-level of signal energies decomposition [18]-[23]. This particular property of Wavelet transform is possible by the advantage of group of filter banks of low-pass and High-pass filters. However, based on Wavelet filter banks, which determine translation and scaling properties of the waveform, there are many types of Wavelet uniqueness known as Mother Wavelets [9], [18]. Therefore, the selection of specific type family Wavelet can be very serious based on Wavelet attribute such as width of frequency window, decaying, symmetry, regularity, orthogonal, biorthogonal, etc.

In this work, Daubechies Wavelets (dbxx) and Haar Wavelet will be used since they are widely used in engineering applications and for matching the properties of tested signal data form [5]. Yet, keeping in mind the shape of wavelet families, orthogonality (such as Daubechies), and biorthogonality (such as biorxx) of Wavelet transform will have a serious impact on testing analysis and results [5], [24]-[27].

\section{COMPUTATION TECHNIQUE}

Ideally, ADCs output waveform is zero deviation from the original waveform. That is, based on signal full scale range and ADC number of bits, the output digital waveform should have the same LSB codes throughout the waveform voltage division [2], [4]. Yet, practically, ADCs digital output signal $\bar{x}(t)$ contains error build into the output waveform (12) due to noise, heat, and other effect that may cause waveform distortion.

$$
\bar{x}(t)=x(t)+e
$$

Where $x(t)=$ Original signal values, and $e=$ error-values

That is, output signal is a combination of both signal data bits and error bits. Some of these errors are due to quantization process which depends on ADCs number of bits that determine quantization step size $(\Delta)$. As in shown Figure 1, the analog input waveform is digitized by sampling and quantization process to provide an output digital waveform as in Figure 2.

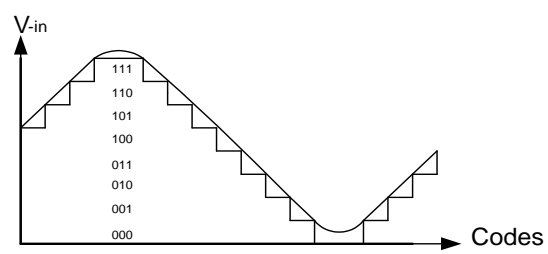

Figure 1. Analog Input Signal 


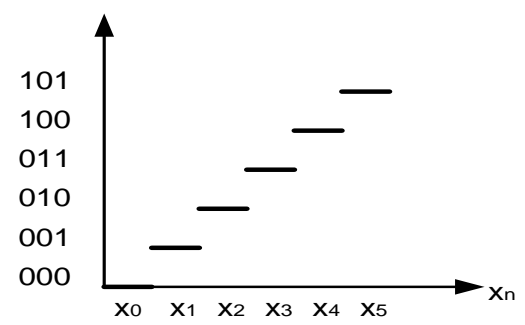

Figure 2. Digital output codes

As a result, ADCs number of bits (n) will affect the size of such a code pattern LSB and any deviation will result in DNL error. In this work, a real time (practical) digital waveform will be simulated as an output signal based on ADC number of bits. The generated digital waveform will be modulated by Hilbert Transform to identify the real and imaginary part of the signal. By capturing the imaginary part, a further process will be conducted by DWT to fetch the tested waveform data by translation and dilation effect through low-pass and high-pass filtersto produce approximation coefficients $\left(s_{n}\right)$ detail coefficients $\left(d_{n}\right)$ as in (13) and (14) respectively.

$$
\begin{aligned}
& {\left[\begin{array}{c}
\vdots \\
s_{n-1,-1} \\
s_{n-1,0} \\
s_{n-1,1} \\
\vdots
\end{array}\right]=\left[\begin{array}{ccccccccc}
\ldots & \ldots & \ldots & & & & & & \\
\ldots & \tilde{h}_{-1} & \tilde{h}_{0} & \tilde{h}_{1} & \tilde{h}_{2} & \ldots & & & \\
& & \ldots & \tilde{h}_{-1} & \tilde{h}_{0} & \tilde{h}_{1} & \tilde{h}_{2} & & \\
& & & \ldots & \tilde{h}_{-1} & \tilde{h}_{0} & \tilde{h}_{1} & \tilde{h}_{2} & \ldots \\
& & & & & & \ldots & \ldots & \ldots
\end{array}\right]\left[\begin{array}{c}
\vdots \\
s_{n,-1} \\
s_{n, 0} \\
s_{n, 1} \\
\vdots
\end{array}\right]} \\
& {\left[\begin{array}{c}
\vdots \\
s_{n-1,-1} \\
d_{n-1,-1} \\
s_{n-1,0} \\
d_{n-1,0} \\
s_{n-1,1} \\
d_{n-1,1} \\
\vdots
\end{array}\right]=\left[\begin{array}{ccccccccccc}
\ldots & \ldots & \ldots & & & & & & \\
\ldots & \tilde{h}_{-1} & \tilde{h}_{0} & \tilde{h}_{1} & \tilde{h}_{2} & \ldots & & & \\
\ldots & \tilde{g}_{-1} & \tilde{g}_{0} & \tilde{g}_{1} & \tilde{g}_{2} & \ldots & & \\
& & \ldots & \tilde{h}_{-1} & \tilde{h}_{0} & \tilde{h}_{1} & \tilde{h}_{2} & \ldots & \\
& & \ldots & \tilde{g}_{-1} & \tilde{g}_{0} & \tilde{g}_{1} & \tilde{g}_{2} & \ldots & \\
& & & \ldots & \tilde{h}_{-1} & \tilde{h}_{0} & \tilde{h}_{1} & \tilde{h}_{2} & \ldots \\
& & & \ldots & \tilde{g}_{-1} & \tilde{g}_{0} & \tilde{g}_{1} & \tilde{g}_{2} & \ldots \\
& & & & & & \ldots & \ldots & \ldots \\
& & & & & & \ldots & \ldots & \ldots
\end{array}\right]\left[\begin{array}{c}
\vdots \\
\vdots \\
\vdots \\
s_{n,-1} \\
s_{n, 0} \\
s_{n, 1} \\
\vdots \\
\vdots \\
\vdots
\end{array}\right]}
\end{aligned}
$$

Then decomposed tested signal data by taking the detail coefficients $\left(d_{n}\right)$ and down sample by half (15) and (16)

$$
\begin{aligned}
& \left(\ldots d_{n-1,-1} d_{n-1,0}, d_{n-1,1}, d_{n-1,2}, d_{n-1,3}, d_{n-1,4}, d_{n-1,5}, d_{n-1,6}, d_{n-1,7}, \ldots\right) \\
& \left(\ldots d_{n-1,-1}, d_{n-1,1}, d_{n-1,3}, d_{n-1,5}, d_{n-1,7}, \ldots\right)
\end{aligned}
$$

To compute instantaneous DNL measurements (17), high-pass coefficients of second level decomposition will be obtained to estimate the difference between the magnitudes of consecutive real codes [1], [2].

$$
\operatorname{DNL}(n)=\frac{\max \left\{|| d_{n-1, j}|-| d_{n-1, j+1}||\right\}}{\Delta_{\text {ideal }}}-1
$$

where $\Delta_{\text {ideal }}$ is ideal LSB [1] 


\section{SIMULATION AND MEASURMENTS}

A range of Analog to Digital Converts was simulated using Matlab simulation software. The range was based on ADCs number of bits (2-20 Bits) to be tested for worst case instantaneous DNL. The generated output signal was modulated by Hilbert Transform to obtain the characteristic of the signal in real and imaginary part as shown in Figure 3.

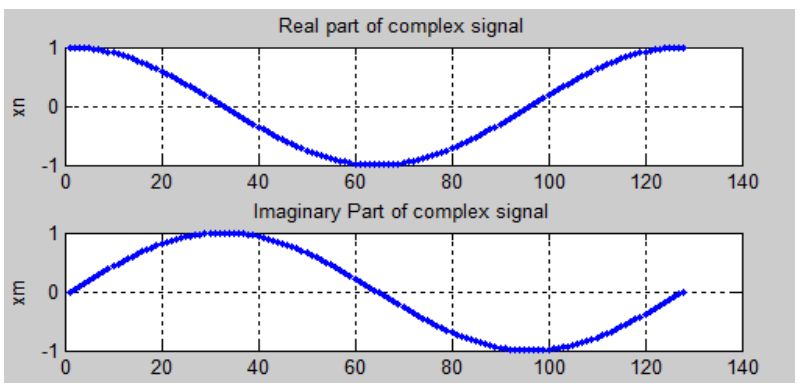

Figure 3. Hilbert Transform of real and imaginary part

Upon processing the signal by Hilbert Transform, the imaginary part of the signal (sinewave) was quantized and converted for sampling Interleave as shown in Figure 4.

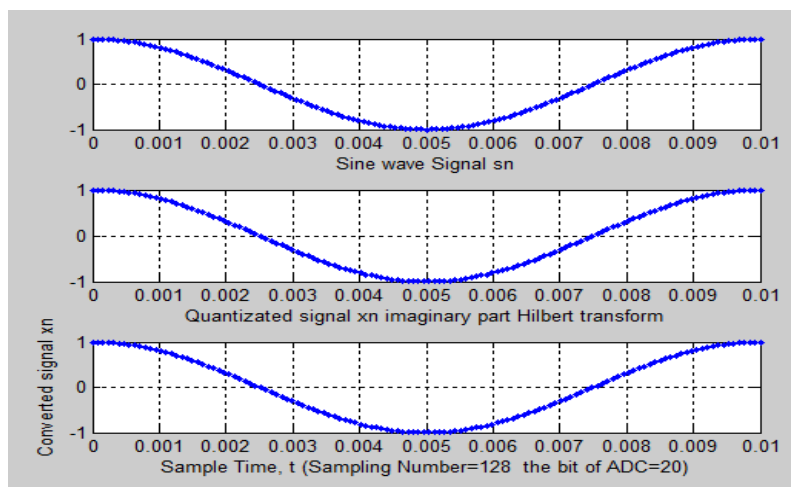

Figure 4. Hilbert Transform imaginary signal part, quantized imaginary part, and sample time

Meanwhile, the imaginary converted part of the waveform was checked by the Fourier Transform algorithms to verify the signal by time and frequency domain as shown in Figure 5.

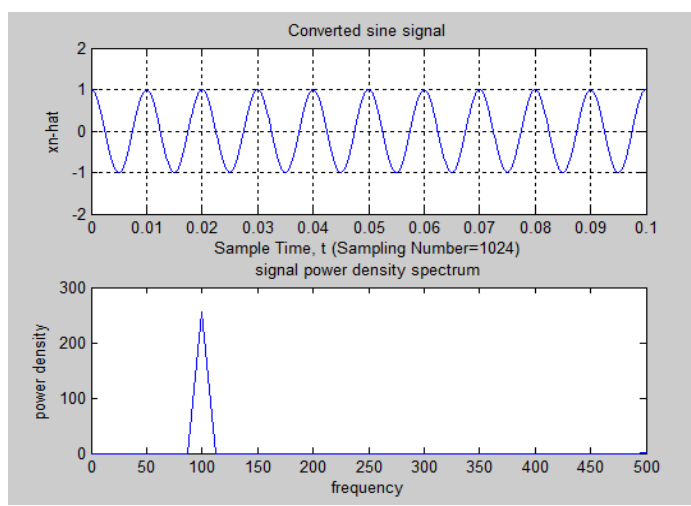

Figure 5. Hilbert converted sinewave signal in time domain and power density

Hilbert Based Testing of ADC Differential Non-linearity Using Wavelet Transform... (Emad A. Awada) 
Based on such algorithms, the quantized Interleave signal data form is acquired for further process of decomposition to suppress the waveform data sample on multilevel as specified in Figure 6 and 7 consecutively for first level and second level DWT decomposition process.

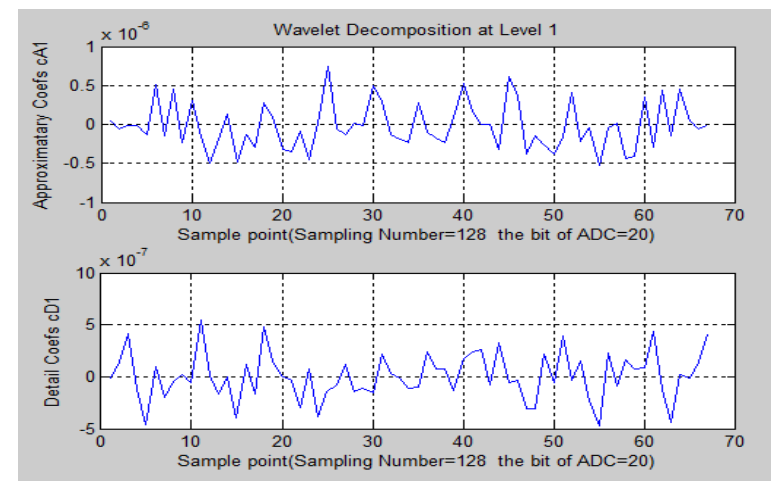

Figure 6. Discrete Wavelet Transform (first level decomposition)

In first level of DWT decomposition, the Interleave signal was decomposed into detail and approximation coefficientby splitting the waveform data into half. Meanwhile, the detail coefficient from first decomposition process was used for a second round of decomposition as in Figure 7. The detail part of the second coefficientwas used to estimate for instantaneous DNL.

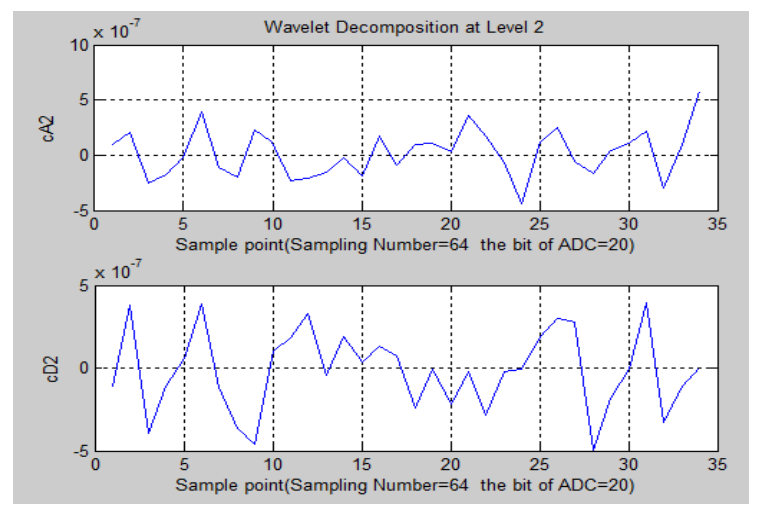

Figure 7. Discrete Wavelet Transform (Second level decomposition)

\section{RESULTS AND DISCUSSION}

In instantaneous testing of digitized output waveform through proposed testing algorithms of Hilbertand Wavelet transform decomposition strategy, it was clear that the new algorithm has shown an enhancement in both testing results and techniques. Using Matlab to simulate various numbers of bits ADCs, and several types of discrete Wavelet, results have shown close approximate to conventional testing method.

In this work, DNL was the focus of testing and verification in term of accuracy with conjunction of short time testing process. That is, as wavelet distinct properties of dilation and translation, a unique type wavelet must be used to give a close approximate match of tested waveform characteristics. For example, generated under test sine-waveform was tested with several types of wavelets. With Haar wavelet characteristics, as shown in Figure 8, the sharp structures edges and straight line of re-adjusting and shifting window did not give close match to tested waveform. 


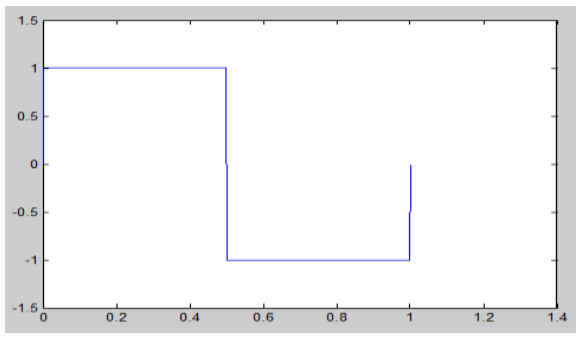

Figure 8. Haar Wavelet

This in return, did not show very accurate result as shown in Tables 1-3. Meanwhile, the Daubechies family had out performed the Haar Wavelet due approximate similarity of the original tested sinewave in pattern as shown in Figure 9.

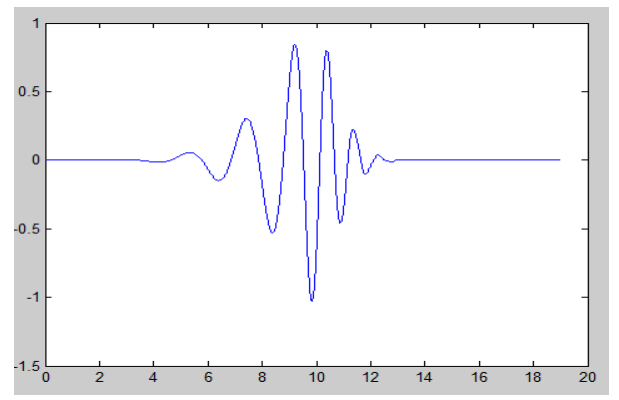

Figure 9. Daubechies family Wavelet

That is, the similarity in waveform pattern allows the Daubechies family wavelet to imitatethe under testing waveform and analyze through adjusted shifted windows with higher detection sensitivity. As a result, instantaneous DNL measurements was obtained and compared as shown in Tables 1- 3 at several testing frequencies.

Table 1. DNL estimation (conventional method FFT Vs. Wavelet based Hilbert Transform 100khz

\begin{tabular}{lcccccccccc}
\hline & 2 Bits & 4 Bits & 6 Bits & 8 Bits & 10 Bits & 12 Bits & 14 Bits & 16 Bits & 18 Bits & 20 Bits \\
\hline HAAR & 0.74 & 0.53 & 0.47 & 0.39 & 0.58 & 0.56 & 0.13 & 0.57 & 0.24 & 0.38 \\
DB2 & 0.56 & 0.34 & 0.39 & 0.37 & 0.37 & 0.33 & 0.31 & 0.31 & 0.35 & 0.26 \\
DB4 & 0.53 & 0.31 & 0.33 & 0.31 & 0.28 & 0.34 & 0.29 & 0.32 & 0.37 & 0.22 \\
DB10 & 0.55 & 0.36 & 0.36 & 0.32 & 0.36 & 0.39 & 0.38 & 0.35 & 0.36 & 0.28 \\
Conv. & 0.49 & 0.44 & 0.31 & 0.31 & 0.33 & 0.38 & 0.39 & 0.32 & 0.38 & 0.28 \\
\hline
\end{tabular}

Table 2. DNL estimation (conventional method FFT Vs. Wavelet based Hilbert Transform 150khz

\begin{tabular}{lcccccccccc}
\hline HDWT/FFT & 2 Bits & 4 Bits & 6 Bits & 8 Bits & 10 Bits & 12 Bits & 14 Bits & 16 Bits & 18 Bits & 20 Bits \\
\hline HAAR & 0.77 & 0.39 & 0.47 & 0.55 & 0.52 & 0.50 & 0.15 & 0.53 & 0.28 & 0.35 \\
DB2 & 0.56 & 0.32 & 0.40 & 0.36 & 0.39 & 0.38 & 0.35 & 0.33 & 0.39 & 0.27 \\
DB4 & 0.50 & 0.30 & 0.32 & 0.23 & 0.24 & 0.36 & 0.27 & 0.23 & 0.50 & 0.36 \\
DB10 & 0.58 & 0.37 & 0.33 & 0.30 & 0.33 & 0.41 & 0.40 & 0.34 & 0.36 & 0.28 \\
Conv. & 0.49 & 0.43 & 0.33 & 0.40 & 0.23 & 0.31 & 0.23 & 0.44 & 0.46 & 0.28 \\
\hline
\end{tabular}

Table 3. DNL estimation (conventional method FFT Vs. Wavelet based Hilbert Transform 200khz

\begin{tabular}{lcccccccccc}
\hline HDWT/FFT & 2 Bits & 4 Bits & 6 Bits & 8 Bits & 10 Bits & 12 Bits & 14 Bits & 16 Bits & 18 Bits & 20 Bits \\
\hline HAAR & 0.77 & 0.23 & 0.47 & 0.35 & 0.52 & 0.50 & 0.15 & 0.53 & 0.28 & 0.35 \\
DB2 & 0.56 & 0.32 & 0.40 & 0.36 & 0.39 & 0.38 & 0.35 & 0.33 & 0.39 & 0.27 \\
DB4 & 0.50 & 0.30 & 0.30 & 0.23 & 0.24 & 0.36 & 0.27 & 0.23 & 0.50 & 0.56 \\
DB10 & 0.58 & 0.36 & 0.33 & 0.30 & 0.33 & 0.41 & 0.40 & 0.34 & 0.36 & 0.28 \\
Conv. & 0.58 & 0.41 & 0.53 & 0.40 & 0.23 & 0.31 & 0.20 & 0.44 & 0.46 & 0.28 \\
\hline
\end{tabular}

On the other hand, using Hilbert Transform and Wavelet decomposition to eliminate unrequired data

Hilbert Based Testing of ADC Differential Non-linearity Using Wavelet Transform... (Emad A. Awada) 
and detection of function peaks, helps reducing the testing duration time significantly. That is, using the imaginary waveform part of Hilbert Transform with the decomposition process of Wavelet, permit the reduction of number of compiled data samples by half at each decomposition level to end-up with nearly one fourth collected sample bits for compiling process.

\section{CONCLUSION}

In this new work of testing Differential Non-Linearity performance for Analog to Digital Converts, Hilbert Transform was implemented as an initial modulation test base. As a result, a particular data was extracted from the original waveform to create platform for Discrete Wavelet Algorithms analysis. Such an algorithm will help reducing the number of waveform data collected by the advantage of both Hilbert and Wavelet transform. That is, less samples to collect and store, shorter compiling process, and accurate error estimation (DNL) near to conventional estimation of Histogram technique.

\section{ACKNOWLEDGEMENTS}

The authors are grateful to Applied Science Private University, Amman - Jordan, for the financial support grated to cover the publication fee of this paper research articles.

\section{REFERENCES}

[1] T. Yamaguchi and M. Soma, "Dynamic Testing of ADCs Using Wavelet Transform", IEEE International Test Conference, pp. 379-388, Nov. 1997.

[2] C. Akujuobi, E. Awada, M, Sadiku, and A. Warsame, "Wavelet-based differential nonlinearity testing of mixed signal system ADCs". IEEE Southeast Con, pp. 76 - 81, Mar 2007.

[3] P. Ramos, J. da Silva, D. Ferreira and M. Santos, "Statistically enhanced analogue and mixed-signal design and test", IEEE 21st International Mixed-Signal Testing Workshop (IMSTW), 2016, pp. 1-5.

[4] Mark Baker, "Demystifying Mixed Signal Test Methods". Newnes, Elsevier Science, pp. 147-237, 2003.

[5] E. Awada \& C. Akuuobi, "DWT Testing of DAC Effective Number of Bits", Proceedings of the IASTED International Conference Circuit and System, pp. 145-150, 2010.

[6] P. Ramos, J. da Silva, D. Ferreira and M. Santos, "Statistically enhanced analogue and mixed-signal design and test", IEEE 21st International Mixed-signal Testing Workshop (IMSTW), pp. 1-5, 2016.

[7] Á. Gómez-Pau, L. Balado, J. Figueras, "Mixed-signal test band guarding using digitally coded indirect measurements", International Conference on Synthesis, Modeling, Analysis and Simulation Methods and Applications to Circuit Design (SMACD), pp. 1-4, 2015.

[8] E. Awada, "The Application Wavelet Transform Algorithm in Testing ADC Effective Number of Bits", International Journal of Computer Science \& Information Technology (IJCSIT), Vol 5, No 5, pp. 161-169, 2013.

[9] E. Awada \& M. Alomari, "Application of Wavelet Transform Analysis to ADCs Harmonics Distortion," Computer and Information Science, Vol. 6, 118-124, 2013.

[10] S.L.M. Hassan, N. Sulaiman, S.S. Shariffudin, T.N.T. Yaakub, "Signal-to-noise Ratio Study on Pipelined Fast Fourier Transform Processor", Bulletin of Electrical Engineering and Informatics, Vol 7, 2018 pp. 230-235

[11] B. Vargha, J. Schoukens, Y. Rolain, "Using reduced-order models in D/A converter testing", IEEE Instrumentation and Measurement Technology Conference, pp. 701-706, May 2002.

[12] W. Chen,Y. Zhu, X. Liu, X. Li, and D. Ou,"Combining the histogram method and the ultrafast segmented model identification of linearity errors algorithm for ADC linearity testing", IEEE European Test Symposium, pp.1-2, 2016.

[13] S. Max, R. Liggiero, "Enhanced ADC sine wave histogram test", IEEE International Instrumentation and Measurement Technology Conference Proceedings, pp. 1652 - 1657, 2015.

[14] J. Domingos, A. Evans, "Digital pseudorandom uniform noise generators for ADC histogram test", IEEE Conference on Design of Circuits and Integrated Systems, pp. 1-6, 2015.

[15] Y. Min, H. Ki Kim, Y. Ri Kang, G. Su Kim, J. Park, S. Kim, "Design of Wavelet-Based ECG Detector for Implantable Cardiac Pacemakers", IEEE Transactions on Biomedical Circuits and Systems, 2013, pp. 426-436.

[16] E. Awada, M. Alomari, E. Radwan, "Wavelet Estimation of THD Based on Hilbert Transform Extraction", International Journal of Applied Engineering Research, pp. 6451-6456, 2016.

[17] S. Abeysekera, "An efficient Hilbert transform interpolation algorithm for peak position estimation", IEEE Statistical Signal Processing, $11^{\text {th }}$ Signal Processing Workshop, pp. 417-420, 2001.

[18] L. Peng, D. Jian-dong, "Characteristic Analysis and Selection of Wavelets Applicable for Ultra-High-Speed Protection", IEEE Transmission and Distribution Conference, pp 1-5, 2005.

[19] A. Mitiche, L. Mitiche, and H. Naimi, "Three levels discrete wavelet transform elliptic estimation for ECG denoising". 4th International Conference on Control Engineering \& Information Technology (CEIT), 2016. pp. 1-5.

[20] S. Mallat “A wavelet tour of signal processing” Academic Press, PP. 220-314, 2001. 
[21] C. Esmeralda, D. Poppi Lodaya, "EEG Based Emotion Monitoring Using Wavelet and Learning Vector Quantization", Proceeding of the Electrical Engineering Computer Science and Informatics, Vol 4, pp.93-103, 2017.

[22] E. Awada, "Analysis of SFDR Using Power Spectrum Based on Wavelet Extraction", International Journal of Applied Engineering Research, pp. 1256-1260, 2016.

[23] Z. Bingyi, C. Haian, "Application of Wavelet Multi-Resolution Analysis to Harmonics Detection Based on MATLAB in power system", IEEE International Conference on Automation and Logistics, pp. 137-143, 2017.

[24] A. Reddy, C. Navitha, K. Sivani, "Performance Evaluation of Adaptive Continuous Wavelet Transform based Rake Receiver for UWB Systems", International Journal of Electrical and Computer Engineering (IJECE), Vol 8, 2018.

[25] Y. Qing-Yu, M. Long-Hu, "Fault Line Detection of Non-Effectively Earthed Neutral System Based onModulus Maximum Determining Polarity", IEEE Conference on Power and Energy Engineering, pp. 1-4, 2009.

[26] Y. Hawwar, A. Reza, "Filtering (Denoising) in the Wavelet Transform Domain", IEEE International Conference, pp. 266-269; 2000.

[27] B. Silverman and J. Vassilicos, Wavelet: The Key to Intermittent Information (New York, NY: Oxford University Press, 2000). 